\title{
RECURSIVE EVALUATION OF SOME BIVARIATE COMPOUND DISTRIBUTIONS
}

\author{
BY \\ RALUCA VERnic \\ "Ovidius" University of Constanta, Romania
}

\begin{abstract}
In this paper we consider compound distributions where the counting distribution is a bivariate distribution with the probability function $\left(p_{n_{1}, n_{2}}\right)_{n_{1}, n_{2} \geq 0}$ that satisfies a recursion in the form

$$
\begin{aligned}
p_{n_{1}, n_{2}}= & \left(a_{0}+\frac{a_{1}}{n_{1}}+\frac{a_{2}}{n_{2}}+\frac{a_{12}}{n_{1} n_{2}}\right) p_{n_{1}-1, n_{2}-1}+\left(b_{0}+\frac{b_{1}}{n_{1}}\right) p_{n_{1}-1, n_{2}} \\
& +\left(c_{0}+\frac{c_{2}}{n_{2}}\right) p_{n_{1}, n_{2}-1}, n_{1}, n_{2}=1,2, \ldots
\end{aligned}
$$
\end{abstract}

We present an algorithm for recursive evaluation of the corresponding compound distributions and some examples of distributions in this class.

\section{KEYWORDS}

Recursions; compound distributions; bivariate distributions: Trinomial distribution, bivariate Negative Binomial, bivariate Poisson.

\section{INTRODUCTION}

Let $N$ denote the number of claims occurring in an insurance portfolio within a given period, and $Y_{i}$ the amount of the $i$ th of these claims. We assume that these claim amounts are positive, integer-valued, mutually independent and identically distributed with the common probability function (p.f.) $f$, and independent of $N$. If $p$ denotes the p.f. of $N$, then the distribution of the aggregate claims $X=\sum_{i=1}^{N} Y_{i}$ is a compound distribution
with p.f.

$$
g=\sum_{n=0}^{\infty} p_{n} f^{* n}
$$


We make the convention that $\sum_{i=a}^{b}=0$ when $b<a$.

Panjer (1981) showed that when $p$ satisfies a recursion in the form

$$
p_{n}=\left(a+\frac{b}{n}\right) p_{n-1}, n=1,2, \ldots
$$

than $g$ satisfies the recursion

$$
g(x)=\sum_{y=1}^{x}\left(a+\frac{b y}{x}\right) f(y) g(x-y), x=1,2, \ldots
$$

Let us now consider that the policies of an insurance portfolio are submitted to claims of two kinds and $N_{i}$ is the random variable (r.v.) yearly frequency of type $i$ claims, $i=1,2$. The distribution of the pair $\left(N_{1}, N_{2}\right)$ is provided by the joint probabilities

$$
p_{n_{1}, n_{2}}=P\left(N_{1}=n_{1}, N_{2}=n_{2}\right), n_{1}, n_{2}=0,1, \ldots
$$

For the compound model, the aggregate amount of claims of the portfolio is $X=X_{1}+X_{2}$ where, for $i=1,2, X_{i}=\sum_{j=1}^{N_{i}} Y_{i j}$ is the aggregate amount of type $i$ claims. In addition to $N_{i}$, this expression needs the sequence $\left(Y_{i j}\right)_{j \geq 1}$ of the amounts of type $i$ claims.

We assume that the following hypotheses are fulfilled (Partrat, 1993): H1: For $i=1,2$ the r.v. $N_{i}$ and $\left(Y_{i j}\right)_{j \geq 1}$ are independent, and the r.v. $N_{i}$ and $\left(Y_{3-i j}\right)_{j \geq 1}$ are independent.

H2: The r.v. $\left(Y_{1 j}\right)_{j \geq 1}$ and $\left(Y_{2 j}\right)_{j \geq 1}$ are independent.

H3: For $i=1,2$ the r.v. $Y_{i j}, j \geq 1$, are independent and identically distributed with the common p.f. $f_{i}(x)=P\left(Y_{i j}=x\right), x=0,1, \ldots$ We assume that $P\left(Y_{i j}>0\right)=1$.

Then the aggregate amounts of claims $\left(X_{1}, X_{2}\right)$ have a bivariate compound distribution with the p.f.

$$
g\left(x_{1}, x_{2}\right)=P\left(X_{1}=x_{1}, X_{2}=x_{2}\right)=\sum_{n_{1}=0}^{\infty} \sum_{n_{2}=0}^{\infty} p_{n_{1}, n_{2}} f_{1}^{* n_{1}}\left(x_{1}\right) f_{2}^{* n_{2}}\left(x_{2}\right),
$$

where $f_{i}^{* n_{i}}$ denotes the $n_{i}$-th convolution of $f_{i}, i=1,2$.

Since Panjer (1981), many recursive algorithms have been derived from compound distributions with univariate counting distribution (see Willmot, 1986; Willmot and Panjer, 1987; Panjer and Willmot, 1992; Sundt, 1992; Willmot, 1993; Hesselager, 1994; and others) and also for bi- and multivariate distributions (see Hesselager, 1996; Ambagaspitiya, 1998; Sundt, 1998a, b, 1999a, b). 
Hesselager (1996) presented some bivariate extensions of Panjer's recursion, using bivariate generalisations of the counting distribution. $\mathrm{He}$ deduced recursions for $g$ in some cases when $p$ can be interpreted as a bivariate extension of Panjer's counting distributions. Sundt (1999a) studied a multivariate generalisation of Panjer's recursion in another direction than Hesselager, assuming that the claim number is one-dimensional, but each claim is an $m$-dimensional random vector. He also discussed Hesselager's recursion in Model A, giving some modifications.

In this paper (section 2), we derive a recursive scheme for the compound distribution (1.1) when the counting distribution satisfies the recursive relation

$$
\begin{aligned}
p_{n_{1}, n_{2}}= & \left(a_{0}+\frac{a_{1}}{n_{1}}+\frac{a_{2}}{n_{2}}+\frac{a_{12}}{n_{1} n_{2}}\right) p_{n_{1}-1, n_{2}-1}+\left(b_{0}+\frac{b_{1}}{n_{1}}\right) p_{n_{1}-1, n_{2}} \\
& +\left(c_{0}+\frac{c_{2}}{n_{2}}\right) p_{n_{1}, n_{2}-1}, n_{1}, n_{2} \geq 1, \\
p_{n_{1}, 0}= & \left(d_{0}+\frac{d_{1}}{n_{1}}\right) p_{n_{1}-1,0}, n_{1} \geq 1, \\
p_{0, n_{2}}= & \left(e_{0}+\frac{e_{2}}{n_{2}}\right) p_{0, n_{2}-1}, n_{2} \geq 1 .
\end{aligned}
$$

If (1.2)-(1.4) together with the initial value $p_{0,0}$ should determine the p.f., then at least two of the probabilities on the right-hand side of (1.2) should have a non-zero coefficient.

In section 3 we present some examples of distributions in class (1.2)-(1.4): Trinomial distribution, bivariate Negative Binomial and bivariate Poisson.

\section{RECURSION FOR THE BIVARIATE COMPOUND DISTRIBUTION}

In this section, we first derive a recursion for $g$ when (1.2) is satisfied, then for the initial values of $g$ when (1.3) and (1.4) are verified. Finally, a link with Panjer's formula is established.

The following theorem gives the recursion for $g$.

Theorem 1. If (1.2) is satisfied, the compound distribution $g\left(x_{1}, x_{2}\right)$ can be evaluated by the following recursive method:

$$
\begin{aligned}
g\left(x_{1}, x_{2}\right)= & \sum_{y_{1}=1}^{x_{1}} \sum_{y_{2}=1}^{x_{2}}\left(a_{0}+a_{1} \frac{y_{1}}{x_{1}}+a_{2} \frac{y_{2}}{x_{2}}+a_{12} \frac{y_{1} y_{2}}{x_{1} x_{2}}\right) f_{1}\left(y_{1}\right) f_{2}\left(y_{2}\right) \\
& \times g\left(x_{1}-y_{1}, x_{2}-y_{2}\right)+\sum_{y_{1}=1}^{x_{1}}\left(b_{0}+b_{1} \frac{y_{1}}{x_{1}}\right) f_{1}\left(y_{1}\right) g\left(x_{1}-y_{1}, x_{2}\right) \\
& +\sum_{y_{2}=1}^{x_{2}}\left(c_{0}+c_{2} \frac{y_{2}}{x_{2}}\right) f_{2}\left(y_{2}\right) g\left(x_{1}, x_{2}-y_{2}\right), x_{1}, x_{2} \geq 1 .
\end{aligned}
$$


Proof: Using (1.2) in (1.1) and the fact that

$$
f_{i}^{* n_{i}}(0)=\left\{\begin{array}{ll}
1, & n_{i}=0 \\
0, & n_{i} \neq 0
\end{array},\right.
$$

we have for $x_{1} \geq 1, x_{2} \geq 1$

$$
\begin{aligned}
g\left(x_{1}, x_{2}\right)= & \sum_{n_{1}=1}^{\infty} \sum_{n_{2}=1}^{\infty}\left[\left(a_{0}+\frac{a_{1}}{n_{1}}+\frac{a_{2}}{n_{2}}+\frac{a_{12}}{n_{1} n_{2}}\right) p_{n_{1}}-1, n_{2}-1\right. \\
& \left.+\left(b_{0}+\frac{b_{1}}{n_{1}}\right) p_{n_{1}-1, n_{2}}+\left(c_{0}+\frac{c_{2}}{n_{2}}\right) p_{n_{1}, n_{2}-1}\right] f_{1}^{* n_{1}}\left(x_{1}\right) f_{2}^{* n_{2}}\left(x_{2}\right) .
\end{aligned}
$$

We will now introduce conditional expectations like e.g. in the proof of Panjer's formula in Sundt (1993). Splitting the sum in (2.3) into three terms, we obtain for the first one

$$
\begin{aligned}
& \sum_{n_{1}=1}^{\infty} \sum_{n_{2}=1}^{\infty}\left(a_{0}+\frac{a_{1}}{n_{1}}+\frac{a_{2}}{n_{2}}+\frac{a_{12}}{n_{1} n_{2}}\right) p_{n_{1}-1, n_{2}-1} f_{1}^{* n_{1}}\left(x_{1}\right) f_{2}^{* n_{2}}\left(x_{2}\right)= \\
& \sum_{n_{1}=1}^{\infty} \sum_{n_{2}=1}^{\infty} E\left[a_{0}+a_{1} \frac{Y_{11}}{x_{1}}+a_{2} \frac{Y_{21}}{x_{2}}+a_{12} \frac{Y_{11} Y_{21}}{x_{1} x_{2}} \mid \sum_{j=1}^{n_{i}} Y_{i j}=x_{i}, i=1,2\right] p_{n_{1}-\mathrm{i}, n_{2}-1} \\
& \times f_{1}^{* n_{1}}\left(x_{1}\right) f_{2}^{* n_{2}}\left(x_{2}\right)=\sum_{n_{1}=1}^{\infty} \sum_{n_{2}=1}^{\infty} \sum_{y_{1}=1}^{x_{1}} \sum_{y_{2}=1}^{x_{2}}\left(a_{0}+a_{1} \frac{y_{1}}{x_{1}}+a_{2} \frac{y_{2}}{x_{2}}+a_{12} \frac{y_{1} y_{2}}{x_{1} x_{2}}\right) p_{n_{1}-1, n_{2}-1} \\
& \times f_{1}\left(y_{1}\right) f_{2}\left(y_{2}\right) f_{1}^{*\left(n_{1}-1\right)}\left(x_{1}-y_{1}\right) f_{2}^{*\left(n_{2}-1\right)}\left(x_{2}-y_{2}\right)= \\
& \sum_{y_{1}=1}^{x_{1}} \sum_{y_{2}=1}^{x_{2}}\left(a_{0}+a_{1} \frac{y_{1}}{x_{1}}+a_{2} \frac{y_{2}}{x_{2}}+a_{12} \frac{y_{1} y_{2}}{x_{1} x_{2}}\right) f_{1}\left(y_{1}\right) f_{2}\left(y_{2}\right) \\
& \times \sum_{n_{1}=1}^{\infty} \sum_{n_{2}=1}^{\infty} p_{n_{1}-1, n_{2}-1} f_{1}^{*\left(n_{1}-1\right)}\left(x_{1}-y_{1}\right) f_{2}^{*\left(n_{2}-1\right)}\left(x_{2}-y_{2}\right)= \\
& \sum_{y_{1}=1}^{x_{1}} \sum_{y_{2}=1}^{x_{2}}\left(a_{0}+a_{1} \frac{y_{1}}{x_{1}}+a_{2} \frac{y_{2}}{x_{2}}+a_{12} \frac{y_{1} y_{2}}{x_{1} x_{2}}\right) f_{1}\left(y_{1}\right) f_{2}\left(y_{2}\right) g\left(x_{1}-y_{1}, x_{2}-y_{2}\right) .
\end{aligned}
$$


For the second term we obtain

$$
\begin{aligned}
& \sum_{n_{1}=1}^{\infty} \sum_{n_{2}=1}^{\infty}\left(b_{0}+\frac{b_{1}}{n_{1}}\right) p_{n_{1}-1, n_{2}} f_{1}^{* n_{1}}\left(x_{1}\right) f_{2}^{* n_{2}}\left(x_{2}\right)= \\
& \sum_{n_{1}=1}^{\infty} \sum_{n_{2}=1}^{\infty} E\left[b_{0}+b_{1} \frac{Y_{11}}{x_{1}} \mid \sum_{j=1}^{n_{1}} Y_{1 j}=x_{1}\right] p_{n_{1}-1, n_{2}} f_{1}^{* n_{1}}\left(x_{1}\right) f_{2}^{* n_{2}}\left(x_{2}\right)= \\
& \sum_{n_{1}=1}^{\infty} \sum_{n_{2}=1}^{\infty} \sum_{y_{1}=1}^{x_{1}}\left(b_{0}+b_{1} \frac{y_{1}}{x_{1}}\right) p_{n_{1}-1, n_{2}} f_{1}\left(y_{1}\right) f_{1}^{*\left(n_{1}-1\right)}\left(x_{1}-y_{1}\right) f_{2}^{* n_{2}}\left(x_{2}\right)= \\
& \sum_{y_{1}=1}^{x_{1}}\left(b_{0}+b_{1} \frac{y_{1}}{x_{1}}\right) f_{1}\left(y_{1}\right) \sum_{n_{1}=1}^{\infty} \sum_{n_{2}=1}^{\infty} p_{n_{1}-1, n_{2}} f_{1}^{*\left(n_{1}-1\right)}\left(x_{1}-y_{1}\right) f_{2}^{* n_{2}}\left(x_{2}\right)= \\
& \sum_{y_{1}=1}^{x_{1}}\left(b_{0}+b_{1} \frac{y_{1}}{x_{1}}\right) f_{1}\left(y_{1}\right) g\left(x_{1}-y_{1}, x_{2}\right) .
\end{aligned}
$$

Analogously, for the third term we get

$$
\begin{aligned}
& \sum_{n_{1}=1}^{\infty} \sum_{n_{2}=1}^{\infty}\left(c_{0}+\frac{c_{2}}{n_{2}}\right) p_{n_{1}, n_{2}-1} f_{1}^{* n_{1}}\left(x_{1}\right) f_{2}^{* n_{2}}\left(x_{2}\right)= \\
& \sum_{y_{2}=1}^{x_{2}}\left(c_{0}+c_{2} \frac{y_{2}}{x_{2}}\right) f_{2}\left(y_{2}\right) g\left(x_{1}, x_{2}-y_{2}\right) .
\end{aligned}
$$

By summing all these terms we obtain the recursive formula (2.1).

In conclusion, $g\left(x_{1}, x_{2}\right)$ can be calculated when all $g\left(z_{1}, z_{2}\right)$ are known for $z_{1}<x_{1}, z_{2}<x_{2}$.

Remark 1. In particular, Theorem 2.2 in Hesselager (1996) is a special case of Theorem 1 in the present paper.

Let us now consider recursions for the initial values:

Theorem 2. Considering the starting value $g(0,0)=p_{0,0}$, then (a) if (1.3) holds, we have the following recursive formula

$$
g\left(x_{1}, 0\right)=\sum_{y_{1}=1}^{x_{1}}\left(d_{0}+d_{1} \frac{y_{1}}{x_{1}}\right) f_{1}\left(y_{1}\right) g\left(x_{1}-y_{1}, 0\right), x_{1} \geq 1 ;
$$

(b) if (1.4) holds, we also have

$$
g\left(0, x_{2}\right)=\sum_{y_{2}=1}^{x_{2}}\left(e_{0}+e_{2} \frac{y_{2}}{x_{2}}\right) f_{2}\left(y_{2}\right) g\left(0, x_{2}-y_{2}\right), x_{2} \geq 1 .
$$


Proof: For $x_{2}=0$ and $x_{1} \geq 1$, using (2.2) and (1.3) in (1.1), we have

$$
\begin{aligned}
g\left(x_{1}, 0\right) & =\sum_{n_{1}=0}^{\infty} p_{n_{1}, 0} f_{1}^{* n_{1}}\left(x_{1}\right) \\
& =\sum_{n_{1}=1}^{\infty}\left(d_{0}+\frac{d_{1}}{n_{1}}\right) p_{n_{1}-1,0} f_{1}^{* n_{1}}\left(x_{1}\right) .
\end{aligned}
$$

Introducing conditional expectations like in the proof of Theorem 1, we obtain

$$
\begin{aligned}
g\left(x_{1}, 0\right) & =\sum_{n_{1}=1}^{\infty} E\left[d_{0}+d_{1} \frac{Y_{11}}{x_{1}} \mid \sum_{j=1}^{n_{1}} Y_{1 j}=x_{1}\right] p_{n_{1}-1,0} f_{1}^{* n_{1}}\left(x_{1}\right) \\
& =\sum_{n_{1}=1}^{\infty} \sum_{y_{1}=1}^{x_{1}}\left(d_{0}+d_{1} \frac{y_{1}}{x_{1}}\right) p_{n_{1}-1,0} f_{1}\left(y_{1}\right) f_{1}^{*\left(n_{1}-1\right)}\left(x_{1}-y_{1}\right) \\
& =\sum_{y_{1}=1}^{x_{1}}\left(d_{0}+d_{1} \frac{y_{1}}{x_{1}}\right) f_{1}\left(y_{1}\right) \sum_{n_{1}=1}^{\infty} p_{n_{1}-1,0} f_{1}^{*\left(n_{1}-1\right)}\left(x_{1}-y_{1}\right) \\
& =\sum_{y_{1}=1}^{x_{1}}\left(d_{0}+d_{1} \frac{y_{1}}{x_{1}}\right) f_{1}\left(y_{1}\right) g\left(x_{1}-y_{1}, 0\right) .
\end{aligned}
$$

We used (2.6) to get the last equality.

Similarly, we obtain the recursive formula for $g\left(0, x_{2}\right)$, based on (1.4).

Assume now that $N_{1}$ and $N_{2}$ are independent with marginal probability functions $q_{n_{1}}$ and $r_{n_{2}}$ that satisfy Panjer's recursions

$$
\left\{\begin{array}{ll}
q_{n_{1}}=\left(\alpha+\frac{\beta}{n_{1}}\right) q_{n_{1}-1}, & n_{1}=1,2, \ldots \\
r_{n_{2}}=\left(\gamma+\frac{\delta}{n_{2}}\right) r_{n_{2}-1}, & n_{2}=1,2, \ldots
\end{array} .\right.
$$

We introduce the marginal probability functions $g_{1}$ and $g_{2}$ of $X_{1}$ and $X_{2}$, which are now independent so that $g\left(x_{1}, x_{2}\right)=g_{1}\left(x_{1}\right) g_{2}\left(x_{2}\right)$.

In the following, we shall show that under assumptions (2.7) the conditions of Theorems 1 and 2 are satisfied, and that using these theorems is equivalent to applying Panjer's recursion on $g_{1}$ and $g_{2}$.

For $n_{1}, n_{2}>0$ we have

$$
p_{n_{1}, n_{2}}=q_{n_{1}} r_{n_{2}}=\left(\alpha \gamma+\frac{\beta \gamma}{n_{1}}+\frac{\alpha \delta}{n_{2}}+\frac{\beta \delta}{n_{1} n_{2}}\right) p_{n_{1}-1, n_{2}-1},
$$

that is, (1.2) is satisfied with

$$
a_{0}=\alpha \gamma, a_{1}=\beta \gamma, a_{2}=\alpha \delta, a_{12}=\beta \delta ; b_{0}=b_{1}=c_{0}=c_{2}=0 .
$$


Insertion in (2.1) gives

$$
\begin{aligned}
g\left(x_{1}, x_{2}\right)= & \sum_{y_{1}=1}^{x_{1}} \sum_{y_{2}=1}^{x_{2}}\left(\alpha \gamma+\beta \gamma \frac{y_{1}}{x_{1}}+\alpha \delta \frac{y_{2}}{x_{2}}+\beta \delta \frac{y_{1} y_{2}}{x_{1} x_{2}}\right) \\
& \times f_{1}\left(y_{1}\right) f_{2}\left(y_{2}\right) g\left(x_{1}-y_{1}, x_{2}-y_{2}\right) \\
= & \left(\sum_{y_{1}=1}^{x_{1}}\left(\alpha+\beta \frac{y_{1}}{x_{1}}\right) f_{1}\left(y_{1}\right) g_{1}\left(x_{1}-y_{1}\right)\right) \\
& \times\left(\sum_{y_{2}=1}^{x_{2}}\left(\gamma+\delta \frac{y_{2}}{x_{2}}\right) f_{2}\left(y_{2}\right) g_{2}\left(x_{2}-y_{2}\right)\right) \\
= & g_{1}\left(x_{1}\right) g_{2}\left(x_{2}\right) .
\end{aligned}
$$

The last equality follows from Panjer's formula.

We also have $p_{n_{1}, 0}=q_{n_{1}} r_{0}=\left(\alpha+\frac{\beta}{n_{1}}\right) q_{n_{1}-1} r_{0}=\left(\alpha+\frac{\beta}{n_{1}}\right) p_{n_{1}-1,0}$, that is, (1.3) satisfied with $d_{0}=\alpha, d_{1}=\beta$. Insertion in (2.4) gives

$$
\begin{aligned}
g\left(x_{1}, 0\right) & =\sum_{y_{1}=1}^{x_{1}}\left(\alpha+\beta \frac{y_{1}}{x_{1}}\right) f_{1}\left(y_{1}\right) g\left(x_{1}-y_{1}, 0\right)= \\
& =\left(\sum_{y_{1}=1}^{x_{1}}\left(\alpha+\beta \frac{y_{1}}{x_{1}}\right) f_{1}\left(y_{1}\right) g_{1}\left(x_{1}-y_{1}\right)\right) g_{2}(0)=g_{1}\left(x_{1}\right) g_{2}(0) .
\end{aligned}
$$

The last equality follows from Panjer's formula. Analogous for (2.5).

By these calculations we have shown that:

(a) When $N_{1}$ and $N_{2}$ are independent with marginal distributions satisfying Panjer's condition, then their joint distribution satisfies (1.2)-(1.4).

(b) Using the algorithms of Theorems 1 and 2 with this counting distribution is equivalent with evaluating the joint p.f. of $X_{1}$ and $X_{2}$ as the product of their marginal probability functions which are evaluated separately by Panjer's recursion.

Therefore, we have a close relation between Theorems 1, 2 and Panjer's recursion.

\section{EXAMPLES OF BIVARIATE DISTRIBUTIONS SATISFYING (1.2)-(1.4)}

The following bivariate distributions are taken from Kocherlakota and Kocherlakota (1992). As an extension of the univariate case, these bivariate distributions are natural candidates for modelling the claim frequency for different risk portfolios. For example, the bivariate Poisson distribution was already used by Picard (1976), Lemaire (1985), Partrat (1993) etc. 
Example 1: Let us consider the following model (Hesselager, 1996): The distribution of $K=N_{1}+N_{2}$ satisfies Panjer's recursion

$$
P(K=k)=\left(a+\frac{b}{k}\right) P(K=k-1), k=1,2, \ldots,
$$

for some constants $a$ and $b$, and the conditional distribution of $N_{1}$ given $N_{1}+N_{2}$ is binomial with

$$
P\left(N_{1}=n_{1} \mid N_{1}+N_{2}=n_{1}+n_{2}\right)=\left(\begin{array}{c}
n_{1}+n_{2} \\
n_{1}
\end{array}\right) \rho_{1}^{n_{1}} \rho_{2}^{n_{2}},
$$

with $\rho_{1}+\rho_{2}=1$. In Theorem 2.1, Hesselager (1996) proved that for this model we have the recursions

$$
\begin{aligned}
& p_{n_{1}, n_{2}}=\rho_{1}\left(a+\frac{b}{n_{1}}\right) p_{n_{1}-1, n_{2}}+a \rho_{2} p_{n_{1}, n_{2}-1}, n_{1} \geq 1, \\
& p_{n_{1}, n_{2}}=\rho_{2}\left(a+\frac{b}{n_{2}}\right) p_{n_{1}, n_{2}-1}+a \rho_{1} p_{n_{1}-1, n_{2}}, n_{2} \geq 1,
\end{aligned}
$$

with $p_{n_{1},-1}=p_{-1, n_{2}}=0$. These are particular cases of (1.2)-(1.4), so that Hesselager's (1996) Theorem 2.2 is a particular case of Theorems 1 and 2.

Thus, for (3.1) we have

$$
\begin{aligned}
& a_{0}=a_{1}=a_{2}=a_{12}=0 ; b_{0}=d_{0}=a \rho_{1} ; b_{1}=d_{1}=b \rho_{1} ; \\
& c_{0}=e_{0}=a \rho_{2} ; c_{2}=e_{2}=0,
\end{aligned}
$$

and for (3.2) we have

$$
\begin{aligned}
& a_{0}=a_{1}=a_{2}=a_{12}=0 ; b_{0}=d_{0}=a \rho_{1} ; b_{1}=d_{1}=0 ; \\
& c_{0}=e_{0}=a \rho_{2} \text { and } c_{2}=e_{2}=b \rho_{2} .
\end{aligned}
$$

Particular case 1. The Trinomial distribution with parameters $\left(m, r_{1}, r_{2}\right)$, $m$ positive integer, $r_{1}, r_{2} \in(0,1)$, has the p.f.

$$
p_{n_{1}, n_{2}}=\frac{m !}{n_{1} ! n_{2} !\left(m-n_{1}-n_{2}\right) !} r_{1}^{n_{1}} r_{2}^{n_{2}}\left(1-r_{1}-r_{2}\right)^{m-n_{1}-n_{2}}
$$

We make the convention that $0 !=1$.

It is easy to verify that (3.3) satisfies the recursive relations

$$
\begin{aligned}
& p_{n_{1}, n_{2}}=\frac{r_{1}}{1-r_{1}-r_{2}}\left(\frac{m+1}{n_{1}}-1\right) p_{n_{1}-1, n_{2}}-\frac{r_{2}}{1-r_{1}-r_{2}} p_{n_{1}, n_{2}-1}, n_{1} \geq 1, \\
& p_{n_{1}, n_{2}}=-\frac{r_{1}}{1-r_{1}-r_{2}} p_{n_{1}-1, n_{2}}+\frac{r_{2}}{1-r_{1}-r_{2}}\left(\frac{m+1}{n_{2}}-1\right) p_{n_{1}, n_{2}-1}, n_{2} \geq 1,
\end{aligned}
$$


that is (3.1)-(3.2) and the condition $\rho_{1}+\rho_{2}=1$ for

$$
\rho_{1}=\frac{r_{1}}{r_{1}+r_{2}}, \rho_{2}=\frac{r_{2}}{r_{1}+r_{2}}, a=-\frac{r_{1}+r_{2}}{1-r_{1}-r_{2}} \text { and } b=(m+1) \frac{r_{1}+r_{2}}{1-r_{1}-r_{2}} \text {. }
$$

Particular case 2. The bivariate Negative Binomial distribution with parameters $\left(\lambda, \theta_{1}, \theta_{2}\right), \lambda>0, \theta_{1}, \theta_{2} \in(0,1)$ has the p.f.

$$
p_{n_{1}, n_{2}}=\frac{\Gamma\left(\lambda+n_{1}+n_{2}\right)}{\Gamma(\lambda) n_{1} ! n_{2} !} \theta_{1}^{n_{1}} \theta_{2}^{n_{2}}\left(1-\theta_{1}-\theta_{2}\right)^{\lambda} .
$$

It is easy to verify that (3.4) satisfies the recursions

$$
\begin{aligned}
p_{n_{1}, n_{2}} & =\theta_{1}\left(1+\frac{\lambda-1}{n_{1}}\right) p_{n_{1}-1, n_{2}}+\theta_{2} p_{n_{1}, n_{2}-1} \\
& =\theta_{1} p_{n_{1}-1, n_{2}}+\theta_{2}\left(1+\frac{\lambda-1}{n_{2}}\right) p_{n_{1}, n_{2}-1} .
\end{aligned}
$$

We have (3.1)-(3.2) under the assumption $\rho_{1}+\rho_{2}=1$ for

$$
\rho_{1}=\frac{\theta_{1}}{\theta_{1}+\theta_{2}}, \rho_{2}=\frac{\theta_{2}}{\theta_{1}+\theta_{2}}, a=\theta_{1}+\theta_{2}, b=(\lambda-1)\left(\theta_{1}+\theta_{2}\right) .
$$

Example 2: The bivariate Poisson distribution, which depends on three positive parameters $\lambda_{1}, \lambda_{2}, \lambda_{3}$, has the p.f.

$$
p_{n_{1}, n_{2}}=e^{-\left(\lambda_{1}+\lambda_{2}+\lambda_{3}\right)} \sum_{k=0}^{\min \left\{n_{1}, n_{2}\right\}} \frac{\lambda_{1}^{n_{1}-k} \lambda_{2}^{n_{2}-k} \lambda_{3}^{k}}{\left(n_{1}-k\right) !\left(n_{2}-k\right) ! k !}, n_{1}, n_{2} \geq 0 .
$$

It satisfies the recursive relations (Partrat, 1993; Hesselager, 1996)

$$
\begin{aligned}
p_{n_{1}, n_{2}} & =\frac{\lambda_{3}}{n_{1}} p_{n_{1}-1, n_{2}-1}+\frac{\lambda_{1}}{n_{1}} p_{n_{1}-1, n_{2}} \\
& =\frac{\lambda_{3}}{n_{2}} p_{n_{1}-1, n_{2}-1}+\frac{\lambda_{2}}{n_{2}} p_{n_{1}, n_{2}-1}, \quad n_{1}, n_{2} \geq 1 \\
p_{0,0} & =e^{-\left(\lambda_{1}+\lambda_{2}+\lambda_{3}\right)}, \\
p_{n_{1}, 0} & =\frac{\lambda_{1}}{n_{1}} p_{n_{1}-1,0}, n_{1} \geq 1 \\
p_{0, n_{2}} & =\frac{\lambda_{2}}{n_{2}} p_{0, n_{2}-1}, n_{2} \geq 1 .
\end{aligned}
$$


Corresponding to (1.2)-(1.4), we have for example for (3.5)

$$
\begin{aligned}
& a_{0}=a_{2}=a_{12}=0, a_{1}=\lambda_{3} ; b_{0}=0, b_{1}=\lambda_{1} ; c_{0}=c_{2}=0 \\
& d_{0}=0, d_{1}=\lambda_{1} ; e_{0}=0, e_{1}=\lambda_{2} .
\end{aligned}
$$

When applying these recursions in Theorem 1, we obtain the same recursions as Hesselager (1996) for the compound distribution.

Remark 2. These examples show that the parameters in (1.2) are not always uniquely determined. Thus one has some freedom to adjust the parametrisation to make the recursions of Theorem 1 more convenient.

\section{ACKNOWLEDGEMENT}

The author is grateful to the referee for his helpful advice in revising the paper.

\section{REFERENCES}

Ambagaspitiya, R.S. (1998). Compound bivariate Lagrangian Poisson distributions. Insurance: Mathematics and Economics 23, 21-31.

Hesselager, O. (1994). A recursive procedure for calculation of some compound distributions. ASTIN Bulletin 24, 19-32.

Hesselager, O. (1996). Recursions for certain bivariate counting distributions and their compound distributions. ASTIN Bulletin 26, 35-52.

Kocherlakota, S. \& Kocherlakota, K. (1992). Bivariate discrete distributions. Marcel Dekker Inc.

Lemaire, J. (1985). Automobile insurance: Actuarial models. Kluwer Publ.

PANJER, H.H. (1981). Recursive evaluation of a family of compound distributions. ASTIN Bulletin 12, 22-26.

Panjer, H.H. \& Willmot, G.E. (1992). Insurance risk models. Society of Actuaries, Schaumburg, Illinois.

Partrat, C. (1993). Compound model for two dependent kinds of claim, XXIVth ASTIN Colloquium, Cambridge.

PICARD, P. (1976). Generalisation de l'étude sur la survenance des sinistres en assurance automobile. Bulletin de l'Institut des Actuaires Francais, 297, 204-267.

SUNDT, B. (1992). On some extension of Panjer's class of counting distributions. ASTIN Bulletin 22, 61-80.

SunDT, B. (1993). An introduction to non-life insurance mathematics. (3. ed.) Verlag Versicherungswirtschaft e.V., Karlsruhe.

SundT, B. (1998a). The multivariate De Pril transform. Research paper 59, Centre for Actuarial Studies, University of Melbourne.

SUNDT, B. (1998b). On error bounds for multivariate distributions. Research paper 60, Centre for Actuarial Studies, University of Melbourne.

SUNDT, B. (1999a). On multivariate Panjer recursions. ASTIN Bulletin 29, 29-45.

SundT, B. (1999b). Multivariate compound Poisson distributions and infinite divisibility. Statistical report 33. Department of Mathematics, University of Bergen.

WILLmot, G.E. (1986). Mixed compound Poisson distributions. ASTIN Bulletin 16, S59-S79. 
Willmot, G.E. (1993). On recursive evaluation of mixed Poisson probabilities and related quantities. Scandinavian Actuarial Journal, 114-133.

Willmot, G.E. \& PANJER, H.H. (1987). Difference equation approaches in evaluation of compound distributions. Insurance: Mathematics and Economics 6, 43-56.

RALUCA VERNIC

Dept. of Mathematics and Informatics

"Ovidius" University of Constanta

124, Bd. Mamaia

8700 Constanta, Romania 